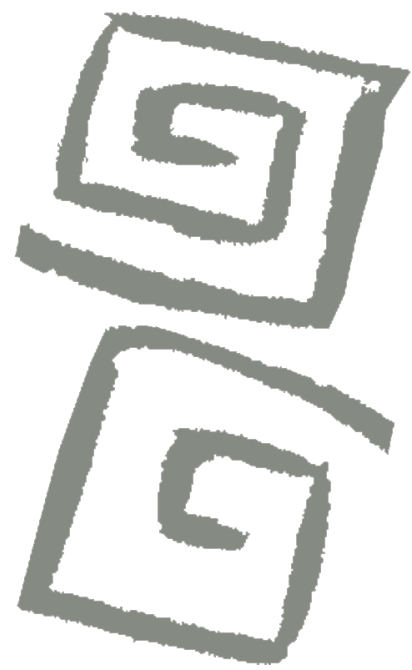

\title{
Abordajes de violencias de género y de interrupción legal del embarazo en servicios de salud durante el aislamiento por COVID-19
}

\author{
Approaches to gender-based violence and legal abortion \\ in health services during COVID-19 lockdown
}

Lorena Saletti-Cuesta', Lila Aizenberg²

'Doctora en Salud, Antropología e Historia. Investigadora adjunta, Centro de Investigaciones y Estudios sobre Cultura y Sociedad, Consejo Nacional de Investigaciones Científicas y Técnicas, Universidad Nacional de Córdoba (CIECSCONICET-UNC), Córdoba, Argentina. $\triangle$ iD

PhD en Sociología. Investigadora adjunta, Centro de Investigaciones y Estudios sobre Cultura y Sociedad, Consejo Nacional de Investigaciones Científicas y Técnicas, Universidad Nacional de Córdoba (CIECSCONICET-UNC), Córdoba, Argentina. $₫$ (iD)
RESUMEN Este estudio buscó indagar cómo los servicios de salud abordan las violencias de género y la interrupción legal del embarazo en contexto de aislamiento social preventivo y obligatorio. Entre mayo y junio de 2020, se realizaron 27 entrevistas telefónicas a profesionales sanitarios e informantes claves de las ciudades argentinas de Córdoba y Resistencia. Se identificaron cambios en la detección, abordaje y demanda vinculados a la reorganización sanitaria, las modificaciones en la relación asistencial y los recursos necesarios para canalizar la demanda. La pandemia ha profundizado los obstáculos de acceso a servicios sanitarios de las mujeres y ha generado otros nuevos. Ante ello, las redes entre profesionales, la práctica sanitaria feminista y los abordajes innovadores fueron identificados como facilitadores de acceso, claves para amortiguar la pandemia y garantizar derechos.

PALABRAS CLAVES Violencia de Género; Aborto; Feminismo; Sector de Atención de Salud; COVID-19; Argentina.

\begin{abstract}
This study is interested in analyzing how health services address genderbased violence and legal abortion in a context of mandatory preventive social isolation. Between May and June 2020, 27 telephone interviews were conducted with healthcare professionals and key informants from Córdoba and Resistencia. Changes in detection, methods, and demand were identified, related to the reorganization of the healthcare system, modifications in the care relationship, and the resources needed to respond to demand. The pandemic has deepened barriers to women's access to health services and has added new ones. Networks among healthcare professionals, feminist healthcare practices, and innovative approaches were identified as facilitators of access, which have been central in mitigating the effects of the pandemic and guaranteeing rights.
\end{abstract}

KEY WORDS Gender-Based Violence; Abortion; Feminism; Health Care Sector; COVID-19; Argentina. 


\section{INTRODUCCIÓN}

Es reconocido que las crisis sanitarias potencian las desigualdades sociales, entre ellas las vinculadas al género ${ }^{(1,2,3)}$. Las medidas de aislamiento y distanciamiento social resultan claves para disminuir el contagio por COVID-19. Sin embargo, se reconoce que aumentan el riesgo a las violencias de género, especialmente dentro de los hogares, ya que aísla a las mujeres con sus agresores, disminuye sus recursos económicos y obstaculiza su acceso a redes y apoyos sociales ${ }^{(4)}$. El aumento de las violencias de género durante la actual pandemia es preocupante a nivel mundial. En Argentina, el incremento de las Ilamadas a la línea telefónica que brinda asesoramiento y contención a mujeres en situación de violencias de género aumentó un $39 \%$ desde el inicio del aislamiento social, preventivo y obligatorio ${ }^{(5)}$. En 2020, también aumentaron un $5,36 \%$ los feminicidios en comparación al año anterior ${ }^{(6)}$. Por todo ello, los gobiernos pusieron en marcha medidas como el fortalecimiento de líneas telefónicas o la excepción de medidas de aislamiento obligatorio en casos de fuerza mayor ${ }^{(7)}$.

Las medidas sanitarias en respuesta a la pandemia afectaron también el acceso a la salud sexual y reproductiva, en general, y al aborto legal, en particular. Se estima que, una vez finalizada la actual pandemia, el porcentaje de mujeres con necesidades insatisfechas de planificación familiar retrocederá entre 20 y 30 años $^{(8)}$, especialmente en los sectores más vulnerables que sufren las mayores desigualdades sociales ${ }^{(9)}$. Las limitaciones en la posibilidad de desplazarse podrían agregar nuevos obstáculos para el acceso a servicios de aborto, retrasando su solicitud y la detección temprana de embarazo.

En Argentina, antes de la sanción, en diciembre de 2020, de la Ley 27610 de Interrupción Voluntaria y Legal del Embarazo, el Art. 86 del Código Penal habilitaba un sistema de permisos o "causales" para la interrupción legal del embarazo (ILE). A pesar del marco regulatorio, las mujeres que han buscado una interrupción legal del embarazo se han enfrentado con distintas barreras que incluían falta de información sobre el alcance de las causales, restricciones ilegales impuestas por el sector salud, límites gestacionales arbitrarios, el requisito de una denuncia u orden judicial para abortar en casos de violación, etc. ${ }^{(10)}$. A pesar de que el gobierno nacional, al inicio de la pandemia, expresó que la interrupción legal del embarazo y otros servicios de salud sexual y reproductiva eran esenciales y emitió recomendaciones específicas para garantizar el acceso, esto no se tradujo en facilitadores concretos para las mujeres ${ }^{(11)}$.

La sobrecarga que impuso la pandemia al sistema de salud agravó las ya deterioradas condiciones de trabajo, la escasez de insumos y de personal, y las barreras de acceso preexistentes ${ }^{(12)}$. El alto dinamismo de la situación epidemiológica y la propia organización del sector salud constituyen un marco de relevancia para preguntarnos cómo ha sido canalizada la atención sanitaria de estas problemáticas. Para ello, indagamos cómo los servicios de salud abordan las violencias de género y la atención de la interrupción legal del embarazo en el contexto de aislamiento social, preventivo y obligatorio en dos ciudades argentinas con altos niveles de contagio de COVID-19 en el periodo estudiado.

\section{METODOLOGÍA}

Se realizó un estudio exploratorio-descriptivo cualitativo en las ciudades de Córdoba y Resistencia. La población de estudio fueron profesionales del primer y segundo nivel de atención e informantes claves de organizaciones sociales, de gestión o centros de asistencia de violencias de género. Los criterios de inclusión fueron: profesionales con mínimo un año de experiencia en su lugar de trabajo, que estén en el momento de la entrevista activos en centros de salud o en hospitales públicos. Informantes claves de ambas ciudades que, al momento del contacto, ocuparan lugares de toma de decisión de política pública, trabajaran en centros de atención de violencias de género o pertenecieran a 
organizaciones sociales vinculadas a violencias de género y/o la ha expresado. Se seleccionó una muestra no probabilística por conveniencia. Las personas entrevistadas fueron captadas de forma voluntaria mediante bola de nieve. La Tabla 1 informa sus características. Durante los meses de mayo a junio de 2020, se realizaron 27 entrevistas semiestructuradas telefónicas de aproximadamente 40 minutos de duración cada una.

A las personas participantes se les informó verbalmente los objetivos y metodología de la investigación y se garantizó el anonimato y confidencialidad. Si bien el proyecto de investigación no fue evaluado por un Comité de ética, dado que no era una exigencia normativa de la entidad financiadora, en todo momento se respetaron las pautas y las normativas éticas vigentes. Una vez obtenido el consentimiento verbal, las entrevistas fueron grabadas y posteriormente transcritas.

Se realizó un análisis temático de los datos, apoyado por el software ATLAS TI 7.5.4, siguiendo la propuesta de Braun y Clarke ${ }^{(13)}$. Este método implicó tres etapas: 1) codificación inicial; 2) organización de códigos en temas y subtemas, y construcción de un primer

Tabla 1. Características sociodemográficas de las personas participantes. Ciudades de Córdoba y Resistencia, Argentina, 2020.

\begin{tabular}{|c|c|c|c|}
\hline Ciudad & Profesión sanitaria & Identidad de género & Lugar de trabajo \\
\hline Córdoba & Psicología & Mujer & Hospital público \\
\hline Córdoba & Enfermería & Mujer & Hospital público \\
\hline Córdoba & Psicología & Mujer & Centro de salud \\
\hline Córdoba & Trabajo social & Mujer & Hospital público \\
\hline Córdoba & Medicina & Hombre & Centro de salud \\
\hline Resistencia & Enfermería & Mujer & Hospital público \\
\hline Córdoba & Enfermería & Mujer & Hospital público \\
\hline Córdoba & Trabajo social & Mujer & Centro de salud \\
\hline Resistencia & Enfermería & Hombre & Centro de salud \\
\hline Córdoba & Medicina & Hombre & Hospital público \\
\hline Resistencia & Bio-imágenes & Hombre & Centro de salud \\
\hline Resistencia & Medicina & Mujer & Hospital público \\
\hline Resistencia & Medicina & Mujer & Centro de salud \\
\hline Resistencia & Psicología & Mujer & Hospital público \\
\hline Córdoba & Psicología & Mujer & Hospital público \\
\hline Córdoba & Medicina & Mujer & Centro de salud \\
\hline Córdoba & Trabajo social & Mujer & Centro de salud \\
\hline Córdoba & Enfermería & Mujer & Centro de salud \\
\hline Córdoba & Enfermería & Mujer & Centro de salud \\
\hline Córdoba & Medicina & Mujer & Hospital público \\
\hline Resistencia & Informante clave & Mujer & Centro de asistencia de violencias de género \\
\hline Resistencia & Informante clave & Mujer & Centro de asistencia de violencias de género \\
\hline Córdoba & Informante clave & Mujer & Organización de mujeres \\
\hline Córdoba & Informante clave & Mujer & Organización de mujeres \\
\hline Córdoba & Informante clave & Mujer & Organización de mujeres \\
\hline Córdoba & Informante clave & Mujer & Gestión/programa de salud provincial \\
\hline Córdoba & Informante clave & Mujer & Organización de mujeres \\
\hline
\end{tabular}

Fuente: Elaboración propia. 
árbol jerárquico; 3) definición de los temas y subtemas con un nivel de abstracción e interpretación mayor. Posteriormente, se realizó un análisis por perfiles, comparando los temas entre ciudades. Finalmente, los resultados fueron revisados con una de las profesionales entrevistadas.

\section{RESULTADOS}

Se identificaron tres temas y subtemas que dan cuenta de la existencia de barreras y facilitadores en la atención a las violencias de género y a la interrupción legal del embarazo en contexto de pandemia.

\section{Cambios en la detección y en la demanda de atención}

Las personas participantes advierten que el contexto de aislamiento social, preventivo y obligatorio expone fuertemente a las mujeres a vivir situaciones de violencias de género y a embarazos no deseados:

...el aislamiento nos encontró a todos, en una situación nueva... y cuando convivís con una persona que te violenta, un contexto de encierro es mucho más agravante y obviamente muchas veces, son forzadas a tener relaciones. (Enfermera, hospital público, Resistencia).

Ante esta situación, coinciden en que el rol del sector salud ante las violencias de género se ha visto modificado, ya que se detectan menos situaciones de violencias de género en comparación al contexto no pandémico, y un recrudecimiento de los casos que llegan a los servicios:

Yo he escuchado que ha aumentado la violencia, que ha habido mayor cantidad de muertes, para colmo nosotros estamos tan enfocados en esto, en no contagiarnos, en que no haya un brote, que uno ha terminado llevando todo a segundo plano. (Enfermera, hospital público, Córdoba)

Ahora no se detectan como en otros momentos. Hoy se resuelve la urgencia y ya se van a la casa. (Médica, centro de salud, Resistencia)

Han ido llegando estas situaciones muy límites... muchas mujeres van al centro de salud cuando llevan a los hijos a la escuela o a la salida/entrada del trabajo, al estar eso limitado, yo lo que creo es que no nos están llegando, por más que sea un centro con una historia, con vínculos" (Psicóloga, centro de salud, Córdoba)

En cuanto a la interrupción legal del embarazo, las personas participantes destacan que ha habido un aumento en la demanda. Uno de los motivos señalados ha sido que el sistema de salud canalizó gran parte de la demanda que antes llegaba a las redes de socorristas (activistas que acompañan y facilitan el acceso al aborto legal), sea por acceso directo de casos o bien por la derivación de las propias socorristas que articularon con el sector salud ante la falta de misoprostol que se dio en el contexto del aislamiento social, preventivo y obligatorio:

La demanda que se ha percibido de las ILE [interrupción legal del embarazo] es también por las socorristas, que son otra grupa de mujeres que también están trabajando y acompañando mujeres... Ellas se han visto más imposibilitadas en estos momentos de trabajar como habitualmente trabajan. (Enfermera, centro de salud, Córdoba)

Además, destacan el problema para abordar casos de interrupción legal del embarazo de mujeres que llegaron con embarazos avanzados. También se dificultaron los controles posabortos en las interrupciones realizadas.

Los siguientes subtemas explican los factores vinculados a los cambios en la detección y en la demanda mencionados. 


\section{Reorganización sanitaria: se desdibujó todo}

La emergencia sanitaria implicó una reorganización del sistema sanitario. En primer lugar, se destaca la modificación en la atención, por ejemplo, los centros de salud y los hospitales abocados a la atención exclusiva de pacientes febriles. También se modificaron los espacios, los modos de circulación dentro de los servicios, y la implementación de nuevos protocolos de atención, entre otros cambios:

De un día para el otro dejamos de atender, porque solo se atendían síntomas febriles. Nosotros estábamos sin saber qué hacer, no entendíamos bien cómo proceder y teníamos que estar sentadas esperando que llegara un posible síntoma febril... esto no es algo que hayamos determinado nosotros, sino que el COE [centro de operaciones de emergencia], que es un grupo de expertos, toma decisiones, que están buenas, pero que no consultan al territorio. (Enfermera, centro de salud, Córdoba)

La modificación de los espacios de atención fue otra barrera que modificó aspectos vinculares claves para el abordaje y detección de violencias de género y el acceso a la interrupción legal del embarazo:

Esto de tener que atender por la ventana, que la gente te tenga que decir todo por la ventana, que nadie pudiese entrar, expulsa. (Trabajadora social, centro de salud, Córdoba)

No podemos hacer una entrevista a fondo, porque yo estoy atendiendo de una ventana. Pidiendo datos. A qué viene. Y no le puedo preguntar de su vida. (Enfermera, centro de salud, Córdoba)

Esta reorganización ha tenido varios impactos mencionados por las personas participantes. Uno de ellos ha sido que las personas usuarias disminuyeron su demanda en los centros de salud y los hospitales, por un lado, por el temor a contagiarse y, por otro, porque estos se han vuelto "expulsivos", restrictivos, sin posibilidad de acceder a consulta a demanda, ni a controles, excepto de urgencia:

...vas al hospital y sabes que en servicio social te van a escuchar... y ahora no podés ingresar al hospital. Entonces la verdad que nos preocupa muchísimo cómo se está resolviendo y creemos que eso va a traer grandes repercusiones de otro tipo; a esta altura no le tengo tanto miedo al COVID como a todo esto otro que hemos dejado de mirar y que sigue pasando. (Trabajadora social, hospital público, Córdoba)

Además, quienes acudían, debían pasar por triajes para acceder a la atención:

Creo que el hospital en este momento ha querido ser tan limpio, tan blanco, que no ha permitido el ingreso de nadie. Persona que ha querido ingresar al hospital y que el hospital ha podido liberarse de ese ingreso ¡lo ha logrado! Entonces, si ha venido una "pacientita" por una violencia de género, realmente no ha llegado a la guardia. Yo no sé si en la misma puerta o en el triaje, porque tienen que pasar por varios lugares, no la han dejado pasar y la han mandado a otro lado. (Enfermera, hospital público, Córdoba)

Así, durante meses, principalmente en hospitales, se ha suspendido la atención de otras problemáticas no vinculadas al COVID-19, como la interrupción legal del embarazo o violencias de género:

Se cancelaron todos los turnos... Ahora en esta situación no nos llega la demanda. Casi todos los días, teníamos situaciones que nos llamaban por personas con razones de suicidios, por razones de violencia... Y la verdad desde que comenzó esta situación no hemos tenido. (Psicóloga, hospital público, Córdoba) 
La gente no quiere ir al hospital porque es el centro de mayor contagio. $Y$ la atención de ILE [interrupción legal del embarazo] estaba centrada en el hospital. Y estábamos viendo de que se pudiera hacer por fuera del hospital, en los centros, pero no sé si se concretó. (Informante clave, centro de asistencia de violencias de género, Resistencia)

La suspensión de controles ginecológicos y los obstáculos para acceder a retirar métodos anticonceptivos ha impactado a su vez en el aumento de los embarazos no deseados:

Un DIU no controlado los últimos dos meses y tuvimos que quitar el DIU, pero en eso ya se había producido un embarazo. (Enfermera, hospital público, Resistencia)

Asimismo, se relató la suspensión de actividades de promoción de la salud y de prevención que habitualmente se realizaban, especialmente en centros de salud, lo que ha modificado sustancialmente el abordaje de las violencias de género:

Lo que nos pasó con la pandemia, que lo que habíamos generado territorialmente se perdió. Porque la gente no se podía juntar en la casa, no podía tener las reuniones con el grupo de mujeres, no podíamos hacer la feria en la plaza, el taller de costura que teníamos acá en el centro de salud, que era un espacio para hablar de violencia... Eso es clave, porque es un espacio de contención, de acompañamiento, de salir de la casa para hacer otra cosa, no lo pudimos tener más. (Trabajadora social, centro de salud, Córdoba)

\section{Relación asistencial: la importancia del vínculo y del cuerpo presente}

La atención en contexto de pandemia ha implicado el uso de elementos de protección, habitar espacios con limitada privacidad, reducir el contacto con pacientes y el tiempo de consulta. Todo ello ha influido en la detección de las violencias de género y en el acceso a la interrupción legal del embarazo puesto que, para su abordaje, se requieren ambientes que faciliten la confianza, el tiempo de escucha, el diálogo y la atención integral:

La situación de entrevista con el barbijo y a veces con la escafandra... son situaciones distantes para poder tener una entrevista, para poder dialogar sobre lo que pasa. Ha sido una distancia más. ¿Viste el guardapolvo del médico que te pone una distancia y una jerarquía? Bueno, imagínate toda una preparación para una entrevista con un bicho que te puede contagiar una persona a la que vos estás queriendo ayudar. (Trabajadora social, centro de salud, Córdoba)

Estos cambios vinculares, el "no poder indagar en profundidad", ha generado también malestares entre profesionales, quienes han cambiado su modo de atención y tareas, principalmente para evitar el contagio:

Ha sido muy distinto porque la atención ha cambiado y... todo ese tipo de problemática suelen salir preguntando un poquito más. Eso se ha dejado de hacer. Es como que la atención ha sido más de guardia. Más expeditiva. Menos integral... En otro contexto con mayor normalidad, se indaga un poquito más y seguramente que esa problemática sale... En este contexto, era más rápido todo. Yo no me podía relajar para hablar, para preguntar un poco más. O sea, me sentía realmente trabajando bajo tensión... Y ahí es difícil. ¡Porque eso depende de nosotras! De que salga. $A$ veces sí, es claro. Vienen por esa necesidad digamos. Esa demanda, y otras veces no, es como tener que buscarlo... Pero ahora, es como todo más rápido, tome, vaya y así... A mí me ha generado mucho malestar. (Médica, centro de salud, Córdoba) 
No es solo colocarle un método anticonceptivo, yo también les pregunto y ellas me cuentan cosas, entonces ese no poder escuchar, no poder charlar, no poder evacuar una duda, que ellas algunas veces dicen: "es una pregunta tonta". No, no es tonta. Porque es una pregunta que a ellas les está ocasionando un miedo. Y poder charlarlo y poder verlo, tranquiliza mucho. Entonces fueron unos meses que se habían perdido todo eso. (Enfermera, centro de salud, Córdoba)

$\mathrm{Si}$ bien las personas participantes indican que han podido reorganizar la atención y realizar seguimientos de situaciones de violencias de género y acompañamientos de la interrupción legal del embarazo mediante videollamadas o llamadas telefónicas, destacan las dificultades que han enfrentado, por ejemplo, para que las mujeres puedan hablar en un espacio de privacidad, y la importancia de, al menos, tener un primer encuentro presencial para establecer relaciones de confianza con las usuarias:

Transitar esas interrupciones en soledad, es muy difícil. Y esta situación de confinamiento, de aislamiento, hace que estemos más solas... muchas de las devoluciones que nos hacen las mujeres es la importancia de tener la presencia, ¿no? Esa mano que sostiene, porque es una situación muy difícil para transitar. Lleva muchísima angustia, estrés, hay que poner el cuerpo, dolores; entonces, no es lo mismo transitarlo en soledad, que alguien que te puede acompañar, sostener, hablar... Yo no creo que haya nada que reemplace lo presencial... está todo, está el cuerpo, está el abrazo, también nos los han quitado... la presencia del otro, ese cuerpo presente, nos los han quitado... al menos para comenzar un espacio terapéutico, tenemos que tener un encuentro presencial... particularmente, en lo que es situación de violencia, creo que es indispensable. Porque muchas veces, incluso, es un cuerpo que sostiene. Porque una característica de la violencia es la pérdida de fuerza: "ya no tengo fuerza", es un cuerpo cansado, agotado, entonces poder también como acompañar y poder levantar ese cuerpo... Por eso te digo la importancia del abrazo, me parece que no hay manera de hacerlo virtual. (Psicóloga, hospital público, Córdoba)

Por otro lado, el vínculo con el barrio, el trabajo con la comunidad, se vio suspendido durante un tiempo, especialmente en los centros de salud clasificados como febriles:

Al ser no febriles, eso nos habilitaba a poder atender algunas cuestiones... pude programar con algunas mujeres del barrio, que tienen merenderos, programar vacunación. Y también para dar la cara, para poder contar: "Che, mirá nos está pasando esto", "estamos trabajando de esta manera" fue todo sobre la marcha. Y fue para nosotros muy aliviador poder estar en el barrio y poder trabajar con la gente y poder volver a dar la cara. Porque lo vincular jes muy importante!... lo habíamos perdido, de un día para el otro, se había ido. Entonces fue muy importante comenzar a hacer este nexo, comenzar a caminar de nuevo al barrio y por más que estábamos con máscaras, con barbijos, con guantes, pero a la gente con escuchar la palabra... llevaba mucha tranquilidad... desde ahí se comenzaron a hacer acciones con las mujeres referentes. (Enfermera, centro de salud, Córdoba)

\section{Recursos limitados o ausentes}

Participantes de Córdoba destacaron la escasez de métodos anticonceptivos: "Estamos con muy pocos recursos. Casi no tenemos métodos, nos vienen de cuentagotas, no tenemos preservativos" (Trabajadora social, centro de salud, Córdoba). Estos faltantes generaron un mayor riesgo de embarazos no deseados: "Los métodos anticonceptivos, los preservativos, las inyecciones por dos o tres 
meses no hubo... los implantes, hace muchísimo que no hay... el DIU, las pastillas... no era algo accesible" (Enfermera, centro de salud, Córdoba).

Destacaron no solo que, durante algunos meses, hubo faltante de misoprostol, sino que, el contexto profundizó los obstáculos derivados de su distribución centralizada:

¡No sabes lo que es! Yo trabajo cerca de [localidad] tener que irnos hasta [barrio de Córdoba], en la otra punta, todo es inaccesible... en mi auto... o sea que pasa más por la voluntad de las personas. Si no es alguien comprometido, olvídate que va hacer toda esa historia para buscar el misoprostol. (Enfermera, centro de salud, Córdoba)

Por otro lado, en ambas ciudades se menciona la falta de elementos de bioseguridad: "Por suerte nos donaron muchos tapabocas, para poder darle a la gente, porque si no, no podés tener una entrevista" (Trabajadora social, centro de salud, Córdoba). Destacan que los elementos de protección recibidos han sido escasos o de mala calidad y que han tenido que comprarlos con sus propios recursos para evitar contagiarse: "Lo que te dan es papel de calcar, jno te cubre nada!" (Enfermero, centro de salud, Resistencia); "tener que comprarnos el barbijo, la cofia, porque no nos provee mucho el Estado" (Médica, centro de salud, Resistencia).

La modificación de la conformación de los equipos, ya sea porque algunas personas se vieron abocadas a nuevas funciones, por reducción del horario presencial, porque eran de riesgo o por los propios contagios en los equipos, fue un obstáculo:

Hubo bajas de gente que garantizaba ILE [interrupción legal del embarazo]... con la pandemia todos los equipos se reestructuraron y a lo mejor quedaban solos (profesionales amigables), entonces muchos avisaron que no iban a poder garantizar ILE... Una situación de violencia sexual, que atendimos... ahí tuvimos que articular, buscar adonde estaban, porque en el medio de la pandemia... habían trasladado la parte del equipo que atendía esa situación... Todo te impacta, también a vos como trabajadora, porque cuando vos entrás a ver con quién armo equipo para acompañar la situación, te van quedando cada vez menos personas. (Psicóloga, centro de salud, Córdoba)

En algunos centros de salud no disponen de profesionales de psicología o trabajo social. Además, reclaman falta de formación en género y de recursos:

Desde el centro de salud solo poner el "cuerpito"... He estado en medio de discusiones entre los varones y las mujeres y ningún otro recurso digamos. Muy pocos recursos... Material ninguno... $Y$ acá no hay ni psicóloga. (Trabajadora social, centro de salud, Córdoba)

Las dificultades para la articulación entre profesionales, profundizadas por el contexto, también fueron destacadas en relación a los centros de atención integral de violencias:

La demanda del [centro de asistencia a las violencias de género] es altísima y el número de profesionales no alcanza a dar respuestas, entonces nos llegan siempre los planteos al hospital... el aislamiento ha profundizado todas las situaciones de malestar, de violencia; la falta de respuesta, porque si bien estamos articulando con el [centro de asistencia] y están dando respuestas... también se han frenado los tratamientos, las asistencias, un poco por la disminución de gente en el [centro de asistencia] y otro por la imposibilidad de las mujeres de llegar a las instituciones. (Trabajadora social, hospital público, Córdoba)

Se fue haciendo el acompañamiento para gestionar la beca, toda la cuestión del alquiler, desde nosotros... con muchas dificultades porque el [centro de asistencia a las violencias de género] 
la atención era por teléfono. (Psicóloga, centro de salud, Córdoba)

El impacto de la ausencia o restricción del servicio de transporte público en el contexto del aislamiento social, preventivo y obligatorio ha sido remarcado:

Este último mes, estamos facilitando... los remises para que Ileguen, porque con esto que no hay transporte, tampoco están pudiendo llegar. (Trabajadora social, hospital público, Córdoba)

...no pueden acceder al hospital, por todo esto del aislamiento y porque también, hoy en día, hace más de tres semanas que el servicio de transporte urbano, no está en funcionamiento y las personas que acceden a la salud pública, muy pocas veces tiene transporte propio. (Enfermera, hospital público, Resistencia).

\section{Vulneración de derechos}

La situación de pandemia ha profundizado las desigualdades sociales existentes. Las dificultades para acceder a la alimentación han sido destacadas:

Al principio, lo que era alimento faltó bastante... hay mucha vulnerabilidad. (Informante clave, centro de asistencia de violencias de género, Resistencia)

...en la pandemia la urgencia que tienen en los barrios es la falta de comida. (Informante clave, organización de mujeres, Córdoba)

Esa gente no tiene ni para comer, entonces, le damos nosotros. Esta pandemia me ha hecho sacar plata de mi bolsillo como nunca antes, porque las mujeres no tienen cómo. Darle $\$ 300$ para que se vaya a la unidad forense y después buscar el botón antipánico... Como equipo de salud hicimos una colecta enorme para comprar bolsones para algunos grupos familiares que no tenían ni qué comer. A ese nivel Ilegamos. (Trabajadora social, centro de salud, Córdoba)

La falta de recursos económicos es un obstáculo clave para mujeres que enfrentan situaciones de violencias de género:

No la querían hacer [denuncia] porque no iban a tener qué comer el otro día... se iban a quedar sin casa, porque el tipo la iba a dejar en la calle... Si no tienen para comer, ¿cómo van a poder pensar que se tienen que separar de este tipo que todos los días la maltrata? (Trabajadora social, centro de salud, Córdoba)

El hacinamiento, la precariedad de las viviendas en contexto de aislamiento social, preventivo y obligatorio, ha aumentado el riesgo de vivir violencias de género y los obstáculos para acceder a la interrupción legal del embarazo:

Estos problemas de consumo, de violencia, todos están como "que la casa es el lugar seguro", por eso te tenés que aislar y ¡la casa no es un lugar seguro para muchas personas! Porque justamente es en la casa donde ocurren todas estas situaciones de violencia, entonces estamos por ahí, recomendando a una persona para que se quede adentro de la casa, para que no se contagie de coronavirus, pero las estas exponiendo a otras problemáticas. (Psicóloga, hospital público, Córdoba)

Estar en la casa todo el tiempo y no poder contarle a alguien. Poder salir con alguna excusa está siendo difícil... Yo creo que al estar en la casa y que esté un montón de gente en la casa es una de las barreras. Como muchas veces, [la interrupción legal del embarazo] se oculta a su pareja, a su suegra, a su madre y de repente estar ahí en el medio, me parece que eso es un problemón. Otra de las cosas que para mí siempre ayuda mucho es cuando nos juntamos con otras y nos 
juntamos a hablar con otras, ahí sale que "Juanita fue a tal lugar" y nos cuentan, y no poder tener ese encuentro, es otra de las barreras. (Informante clave, organización de mujeres, Córdoba)

A esa situación se suman las desigualdades en las tareas de cuidado que recaen especialmente en las mujeres:

Yo lo que he visto es a muchas mujeres expresar el cansancio que es tener al hombre y a los niños todo el día en la casa... te ponen la cara y te dicen: "No aguanto más". (Trabajadora social, centro de salud, Córdoba)

Las desigualdades para acceder a Internet, el no disponer de computadoras o celulares, ha impactado en el acceso a la atención sanitaria, en el vínculo profesionales-usuarias y en el acceso a las escasas políticas sociales de emergencia:

Yo me he sentado tardes enteras con personas a hacer trámites virtuales... Acceso a políticas redistributivas, como el IFE y demás, todo bien, mientras vos tengas cierto nivel de escolaridad y alfabetización... es recontra complicado, hacer cualquier gestión en el ANSES [Administración Nacional de la Seguridad Social] o acá en el registro de la provincia ¡Todo virtual!... un montón de gente ha perdido esos recursos por no saber cómo manejarse en la virtualidad o por no saber abrir el mail ¡Por no tener Internet! (Trabajadora social, centro de salud, Córdoba)

Por último, resalta el impacto del aislamiento social, preventivo y obligatorio en los trabajos precarizados y en la salud:

Esta señora es la quinta vez que viene y no tiene molestias, claramente hay algo. ¿Y vos qué crees? "Bueno doctora, es esto, es este contexto y no tengo trabajo, y no puedo ir a trabajar". Sobre todo, las empleadas domésticas y que con el
IFE no alcanza, y lloran, nervios y sale obviamente. (Médica, centro de salud, Córdoba)

\section{Facilitadores de acceso}

Los obstáculos señalados y el gran impacto de la pandemia en las condiciones laborales del personal de salud (recorte de horas y salarial, precariedad laboral, falta de información y escasa incidencia en las decisiones, no poder acceder a licencias, cansancio, etc.) estuvieron acompañados por el alto compromiso con su rol y sus tareas:

Se demostró la precariedad de la salud pública en el Chaco, estaba muy abandonada. No hubo muchas inversiones estos últimos años. Imagínate hace nueve años que estoy y no soy planta permanente todavía ¿entendés? Y recién ahora se dieron cuenta que yo estoy en el sistema. Me tienen de acá para allá, cubriendo. Ahí se dan cuenta lo importante que somos. (Enfermero, centro de salud, Resistencia)

Este tema profundiza sobre dos aspectos que han facilitado los abordajes y el acceso: las prácticas feministas y los abordajes innovadores.

\section{Redes, compromiso y práctica feminista en salud}

La formación en salud desde una perspectiva de género es clave para modificar las prácticas profesionales:

Nosotros venimos de instituciones formadoras, pero no nos forman para mirar a la persona de manera integral, entonces cuesta mucho aprender. Hay profesionales que hacemos un clic y hay otros que no. Quizás pasan su vida así, su vida profesional sin mirarlo, o porque no quieren o porque cuesta. (Enfermera, centro de salud, Córdoba) 
Destaca el interés y el compromiso personal que ha llevado a varias profesionales a formarse en esta perspectiva con sus propios recursos económicos:

Todas las capacitaciones que nosotros podamos tener sobre género son porque los profesionales hemos decidido, personalmente, hacer capacitaciones sobre género... nos capacitamos y entendemos de la importancia de saber sobre violencia de género. Pero nunca una política que nazca desde el Estado, o desde el Ministerio de Salud o desde el propio hospital. (Enfermera, hospital público, Resistencia)

Algunas de ellas mencionan que no solo es la formación lo que modifica sus prácticas sino tomar consciencia de las desigualdades de género que ellas mismas atraviesan, como las situaciones de violencias de género:

Las formaciones siempre te cambian. Pero lo que más te cambia es cuando te atraviesa el cuerpo... A mí me cambió un montón la forma de intervenir a partir de que yo me vi atravesada, en el cuerpo, por un montón de cuestiones más personales que obviamente uno lo resignifica con la teoría. En nuestras generaciones no venimos formateadas con el feminismo. Nos tuvimos que formatear nosotras... $Y$ yo a partir de verme a mi atravesando algunas situaciones, que me llevaron a separarme de mi expareja y trabajar ese año con un grupo de mujeres muy atravesadas en el cuerpo, por situaciones de violencia re-extremas... Bueno, escucharlas, vivir con ellas... me permitió resignificar un montón de cosas que yo venía viviendo... Después, en estos últimos años, trabajar con las ILE, también. (Trabajadora social, centro de salud, Córdoba)

Formar parte de la Red de Profesionales de la Salud por el Derecho a Decidir ha sido clave en este contexto porque ha facilitado la circulación de información, las articulaciones entre profesionales y les ha brindado apoyo, especialmente en Córdoba:

Nos vamos ayudando. Yo recién pedí las notas para pedir el misoprostol, de una compañera que es de la red y me mando por WhatsApp, cómo formular la nota. Esas cosas así: ¡Todo el tiempo!" (Trabajadora social, centro de salud, Córdoba)

Primero que nada, la formación y la información permanente que circula $y$, por otro lado, es un medio mucho más rápido y de mayor accesibilidad a la respuesta. Tenemos un recursero [guía con recursos de información] que es armado por la red... cuáles son los compañeros que pueden dar respuesta para no estar mandando a las mujeres a que tengan respuestas negativas o que le vulneren los derechos. La manejamos vía WhatsApp. Te llega la demanda, lo pones en el grupo y en algún momento te contestan o llamas a algún referente y es mucho más simple, que a lo mejor haciéndolo a nivel institucional. (Trabajadora social, hospital público, Córdoba)

La verdad que fue muy intenso, muy angustiante al principio, con mucho miedo, la angustia de tener que reorganizar todo a contrarreloj y sin líneas claras desde arriba. Y bueno las redes, se reactivan en los momentos difíciles y nos sostienen. (Psicóloga, centro de salud, Córdoba)

Además, algunas personas mencionaron que la red se vio fortalecida en este contexto, entre otros factores, por la propia necesidad de resolver casos no canalizados:

Nos ayudó a fortalecernos con las redes. Porque nos hemos tenido que comunicar mucho más, porque hemos tenido que hablar muchas más veces, no solamente en capital sino con los compañeros del interior. (Trabajadora social, centro de salud, Córdoba) 
El apoyo de la Red fue clave en este contexto, donde como en otros momentos, han enfrentado resistencias de otros profesionales para garantizar la interrupción legal del embarazo:

También pasa cuando hablamos de ILE y de salud sexual reproductiva, recibimos muchos golpes a quien habla del tema y a quien lo lleva, quien lo discute. (Enfermera, centro de salud, Córdoba)

Vos sabrás que no todos están de acuerdo con esta libertad de poder decidir. Como nosotras tenemos en el turno tarde un pensamiento más abierto, tratamos de guiarla y apoyarla. Le brindamos todas las herramientas. (Enfermera, centro de salud, Córdoba)

En ese mismo sentido, advierten diferencias entre sus prácticas "amigables", como las Ilaman, y la de algunos de sus pares:

La verdad que el centro de asistencia a la víctima de violencia es quien mejor coordina estos casos. Porque nosotros somos un verdadero desastre. Yo he visto cada barbaridad. Cada vez que estoy de guardia trato de ocuparme, porque yo soy una persona muy sensibilizada con la violencia de género, con la $I L E$, porque pasa que siento todo lo que hago por los derechos sexuales, por los derechos humanos, por el derecho de las mujeres. Pero no todos trabajamos así... yo veo las falencias. (Médica, hospital público, Resistencia)

Este compromiso con garantizar derechos lleva a algunas profesionales a definir su práctica como feminista, definiéndola como:

Primero escuchamos a las mujeres, le creemos, y a partir de ello, vemos cómo damos respuestas... ¡Escuchar, creer! Y en función de eso, que la práctica sea una respuesta a la demanda que nos hacen las personas para la cual trabajamos. (Trabajadora social, hospital público, Córdoba)
Por otro lado, se menciona el rol clave que las mujeres han desempeñado en los COmedores comunitarios y cómo estos se han transformado en espacios de encuentro y de abordajes de diversas problemáticas, entre ellas violencias de género y la interrupción legal del embarazo:

En este contexto de pandemia, la referencia han sido los comedores... La demanda concreta ha sido la cuestión de alimentos, en función a eso uno empezó a identificar otras cosas. Que no es solamente esa copa de leche, ese plato de comida, sino que se combina con otra cosa. (Informante clave, organización de mujeres, Córdoba)

\section{Abordajes innovadores}

Frente a los obstáculos han surgido abordajes que han permitido responder a las necesidades. Destacan las acciones realizadas para mantener el vínculo con la comunidad, como el uso de las consultas telefónicas o las videollamadas para abordar situaciones de violencias de género, realizar seguimientos o acompañamientos de la interrupción legal del embarazo. También se fortalecieron diversos canales de comunicación con la comunidad, como la radio comunitaria, la creación de grupos de WhatsApp o las visitas domiciliarias/barriales:

...las veces que hemos salido, hemos dejado carteles, hemos conversado con las mujeres de los merenderos, de que, en caso de violencia, en caso de necesitar una interrupción, en caso de métodos anticonceptivos, que por favor se sigan comunicando, que seguimos estando. (Enfermera, centro de salud, Córdoba)

Disponer de líneas gratuitas para la atención de la salud sexual y reproductiva y para violencias de género, y la novedosa posibilidad de realizar denuncias por esa vía, fue clave: 
Lo que sí he notado es que la línea del 0800 está funcionando realmente ahora... las pacientes así se están animando y están ingresando a través de la línea a los pasos para la ILE. (Médica, hospital público, Resistencia)

Algo que de alguna manera ha facilitado la atención a las mujeres es la atención vía telefónica al 0800 que mejoró mucho, porque todo el grupo de asistencia del [centro de asistencia a las violencias], pasó al 0800. Tener un número gratuito que las mujeres puedan Ilamar... y que tomaran la denuncia también telefónicamente es importante, eso ayudó. (Trabajadora social, centro de salud, Córdoba)

En Resistencia, recalcaron la creación de un sistema de registro informatizado de casos de violencias de género que facilitó el trabajo en red:

Armamos una plataforma virtual a partir del COVID, donde se registran las usuarias asentadas en el 137, que es la línea de emergencia acá en el Chaco... la idea de la plataforma es como una conexión de varios equipos... que se conecten en un mismo eje, en una planilla, y podamos entre todos compartir la información, ver cómo articular (Informante clave, centro de asistencia de violencias de género, Resistencia)

También se mencionó la importancia de conocer a la población mediante georeferenciación:

Lo que hicimos inicialmente fue todo un relevamiento enorme, en todos los barrios donde es nuestra área programática... cosa de tener a los grupos más vulnerables en esta pandemia, tenerlos en el mapa, pero tenerlos en la cabeza. Lo mismo que hacemos con alguno con violencia. (Trabajadora social, centro de salud, Córdoba)
Por último, en Córdoba, al momento de las entrevistas, fue creado un programa de Salud Integral de la Mujer que aspira a ampliar el acceso a métodos anticonceptivos y la asistencia a violencias de género. Sin embargo, las personas participantes no lo conocían.

\section{DISCUSIÓN}

En Argentina, a pesar de que se haya proclamado la salud sexual y reproductiva como un servicio esencial, y de que se haya fortalecido el abordaje de las violencias de género, la pandemia colocó a las mujeres y personas gestantes en una situación de particular riesgo. Este estudio, al igual que otros a nivel nacional e internacional ${ }^{(4,8,12)}$, aporta evidencia de cómo la pandemia profundizó las desigualdades sociales preexistentes, produciendo exclusión social y limitaciones específicas en términos de accesibilidad a servicios sanitarios ${ }^{(14,15)}$. En concreto, el impacto diferencial se observó a partir de marcadores sociales asociados al género y a la clase social, los cuales vienen históricamente articulando y produciendo exclusión social y limitaciones específicas en términos de accesibilidad a servicios sanitarios $^{(14,15)}$. El contexto de aislamiento social, preventivo y obligatorio generó dinámicas particulares, producto de la situación de pobreza y vulnerabilidad que se vio incrementada y que repercutió en las posibilidades de las mujeres de acceder a servicios de protección o denuncia en casos de violencias de género o bien de atención sanitaria ${ }^{(16)}$, tal como lo demuestra este estudio.

Los resultados obtenidos dan cuenta de que esta pandemia modificó el rol de los servicios de salud ante el acceso a la interrupción legal del embarazo y el abordaje de las violencias de género. En términos de la interrupción legal del embarazo, en concordancia con otros estudios internacionales, este trabajo evidenció que la pandemia profundizó barreras de acceso preexistentes y generó otras nuevas, que impactaron desproporcionalmente en los sectores más desfavo$\operatorname{recidos}^{(17,18,19)}$. En sintonía con la bibliografía, 
los resultados obtenidos dan cuenta de una multiplicidad de barreras de acceso a servicios de salud sexual y reproductiva asociadas a la restricción en los desplazamientos, al retraso o rechazo en el uso de servicios por temor al contagio y a la limitación de insumos y servicios específicos ${ }^{(9,20)}$. Por ejemplo, en el Estado español se profundizaron inequidades territoriales ya que, a pesar de que el aborto fue considerado un servicio esencial, la posibilidad de evitar el desplazamiento que supone el asesoramiento inicial, obligatorio por ley, y que sea realizado de forma telemática, fue puesto en marcha solo en Galicia y $\mathrm{Ca}$ taluña ${ }^{(19)}$. Además, se observó una profundización de los obstáculos que históricamente dificultaban el acceso a la prestación de servicios de aborto legal en el sistema de salud y que son propios de la cultura institucional y/o de barreras impuestas por las y los profesionales a través de sus prácticas y actitudes frente al aborto ${ }^{(18)}$. En ese sentido, se observó el impacto de la pandemia sobre la organización del sistema de salud, tanto en la provisión de servicios como del personal disponible y capacitado para brindar atención. Si bien el aislamiento social ha llevado a una reducción general de la demanda de servicios de salud sexual y reproductiva, como también han evidenciado otros trabajos a nivel internacional ${ }^{(19,21,22)}$, nuestros resultados destacan el aumento de la demanda de servicios de aborto en el sector salud, probablemente como resultado de la articulación de profesionales y redes de socorristas y la reciente aprobación de la ley en nuestro entorno ${ }^{(23)}$.

En el contexto de recrudecimiento de situaciones de violencias de género y de aumento exponencial de casos, demostrado en numerosos países ${ }^{(4,5,6,24,25,26,27)}$, este trabajo acompaña a aquellas investigaciones que han señalado las dificultades de detección y atención de casos de violencias de género en el sector salud, producto del propio aislamiento, de reducción del contacto, de la reorganización sanitaria, la falta de protocolos específicos de atención y de espacios de privacidad y contención adecuados que posibiliten generar vínculos de confianza $^{(28,29,30)}$. Algunos de estos obstáculos ya fueron identificados con anterioridad en nuestro contexto ${ }^{(31)}$. Por ejemplo, un estudio cualitativo realizado en Holanda, también destaca el impacto de la reorganización sanitaria en la menor detección de las violencias de género por parte de profesionales, vinculado a la reducción del contacto personal con las usuarias ${ }^{(30)}$. En ese sentido, el rol y el compromiso de las y los profesionales de la salud resulta crucial. Especialmente, porque la pandemia también modificó cómo las mujeres solicitaron ayuda a los servicios profesionales, profundizando las dificultades ya existentes $^{(28,30,32)}$. Una revisión ${ }^{(28)}$ señala que cerca del $90 \%$ de las mujeres que vivieron violencia durante las medidas de aislamiento social por COVID-19 no solicitaron ayuda a las autoridades ni reportaron el abuso sufrido, sobre todo aquellas que vivieron abuso emocional, y accedieron menos a la atención hospitalaria a pesar de que la severidad de las lesiones fue mayor. Cabe señalar que entre las diferentes formas de violencia (psicológica, sexual, económica, etc.) disminuyeron los episodios de agresión física, aunque la gravedad de las agresiones aumentó(28), tal como fue señalado por las personas entrevistadas en este estudio. Asimismo, si bien la atención virtual permitió acceder a los servicios, las barreras identificadas en nuestro contexto, tales como la dificultad de algunas mujeres para hablar en un espacio de privacidad o las desigualdades para acceder a Internet, también han sido señaladas en otros países como Holanda ${ }^{(30)}$ o EEUU ${ }^{(29)}$.

Por otro lado, indagar cómo la pandemia afectó el modo de trabajo de profesionales de sanidad resulta clave para optimizar la atención ${ }^{(30)}$. Los resultados aquí analizados no evidencian, como otros trabajos ${ }^{(33)}$, el recrudecimiento de las inequidades de género preexistentes a la pandemia que atraviesan a los equipos de salud y que afectan en particular a las profesionales mujeres. No obstante, pone en evidencia junto a estos estudios la importancia de abordar la experiencia de profesionales de la salud en pandemia desde una perspectiva de género. En el marco de la ruptura de las redes de apoyo, producto de la situación de aislamiento social, preventivo 
y obligatorio(15) ${ }^{(1)}$ se identificaron distintos elementos que actuaron como facilitadores en la accesibilidad de servicios de aborto legal y en el abordaje de violencias de género que forman parte de una "práctica sanitaria feminista"(34). Entendemos que dicha práctica podría constituirse en un elemento distintivo del contexto argentino que responde a la presencia de sanitarias insertas en redes de apoyo profesional preexistentes a la pandemia que lograron ser catalizadores de una demanda inconclusa y/o no resuelta durante la crisis sanitaria. En el marco de la pandemia, las redes actuaron de manera virtual o telefónica, y no solo lograron mantenerse, sino que se expandieron, amortiguando el impacto de la suspensión de los servicios sanitarios. Si bien es reconocido que las sanitarias, a pesar de los obstáculos vinculados a una organización laboral androcéntrica tienen un estilo de práctica diferencial respecto de sus compañeros ${ }^{(35)}$, pareciera que a estas características claves para abordar violencias de género ${ }^{(36)}$ se le añade un compromiso particular con los derechos de las mujeres. Ese plus feminista identificado en las prácticas profesionales en este estudio, no solo parte del reconocimiento de las desigualdades históricas a las que se enfrentan las mujeres, sino que busca facilitar y fortalecer la igualdad de oportunidades y el disfrute de derechos, a la par que reducir las jerarquías y el distanciamiento entre profesionales y quienes consultan, reconociéndose en muchos aspectos y vivencias iguales a sus usuarias. Estas características han sido vinculadas con una ética feminista en las prácticas asistenciales, identificadas también entre trabajadoras sociales ${ }^{(37)}$ y se vinculan con el impacto que el movimiento de mujeres tiene en la atención a la salud ${ }^{(34)}$. Los aportes del feminismo a la salud son reconocidos ${ }^{(38)}$.

Este estudio dio cuenta de cómo la crisis sanitaria y la emergencia por el aumento social de casos de violencias de género permitió, en algunos casos, una revisión de los canales de atención tradicionales y una planificación de estrategias innovadoras en el contexto del aislamiento social, preventivo y obligatorio -como plataformas virtuales únicas de registro de casos- que facilitaron los abordajes. La implementación de nuevas estrategias y el fortalecimiento de la virtualidad para los abordajes de las violencias de género ha ocurrido también en otros contextos como el holandés ${ }^{(30)}$ o el español ${ }^{(39)}$ donde, al igual que en Argentina ${ }^{(5,25)}$, las llamadas a la línea gratuita de atención de violencias de género aumentaron durante el confinamiento $^{(40)}$.

Entre las limitaciones del estudio destacamos la sobrerrepresentación de una de las ciudades, desde donde se realizó el estudio, y que el método de captación de la muestra puede haber sesgado la inclusión de participantes más comprometidas con el feminismo. En futuros estudios se recomienda indagar el impacto de la aprobación de la Ley 27610 de Interrupción Voluntaria y Legal del Embarazo en el sector salud y los abordajes de las problemáticas estudiadas en contextos de medidas preventivas más flexibles, como el distanciamiento social. Asimismo, se recomienda la inclusión de la perspectiva de las mujeres usuarias de los servicios de salud para indagar sus experiencias durante la pandemia.

\section{CONCLUSIÓN}

Este trabajo es pionero en indagar los abordajes del sector salud frente a las violencias de género y acceso a la interrupción legal del embarazo en contexto aislamiento social, preventivo y obligatorio. Los resultados han evidenciado una mayor demanda de la interrupción legal del embarazo y una menor detección y abordaje de violencias de género en el sector salud, modificando su rol. Se evidencian nuevos obstáculos que, en ocasiones, profundizan los ya existentes. Sin embargo, este contexto brindó oportunidades de repensar la atención y generar procesos de transformación capaces de superar las barreras de acceso a la salud. Destaca el fortalecimiento de prácticas profesionales feministas preexistentes que amortiguaron el impacto desigual de la pandemia sobre las mujeres y 
fueron claves para reducir el impacto de la suspensión de los servicios y abordar la salud. Ante un contexto de incertidumbre prolongada, donde las medidas para enfrentar la pandemia se modifican según la variable situación epidemiológica, destaca la necesidad de políticas públicas que acompañen las prácticas feministas sanitarias identificadas $y$, sobre todo, que fortalezcan el rol del sector salud en la garantía y el disfrute de derechos.

\section{FINANCIAMIENTO}

Esta investigación fue financiada por el Fondo para la Investigación Científica y Tecnológica (PICT 2016-0475).

\section{AGRADECIMIENTOS}

A todas las personas que, en contexto de extrema dificultad, han participado en las entrevistas compartiendo sus experiencias. A Victoria por su excelente trabajo de desgrabación y a Yanina por su amorosa lectura y devoluciones. A la organización Católicas por el Derecho a Decidir-Argentina, que nos apoyó en la realización de esta investigación.

\section{CONFLICTO DE INTERESES}

Las autoras declaran no tener vínculos o compromisos que condicionen lo expresado en el texto y que puedan ser entendidos como conflicto de intereses.

\section{REFERENCIAS BIBLIOGRÁFICAS}

1. Davies E, Bennett E. A gendered human rights analysis of Ebola and Zika: locating gender in global health emergencies. International Affairs. 2016;(92):1041-1060.

2. Onyango MA, Resnick K, Davis A, Shah RR. Genderbased violence among adolescent girls and young women: A neglected consequence of the West African Ebola outbreak. In: Schwartz D, Anoko J, Abramowitz S, eds. Pregnant in the time of Ebola: Global maternal and child health (Medical, Anthropological, and Public Health Perspectives). New York: Springer, Cham; 2019. p. 121-132.

3. European Commission's Humanitarian Aid and Civil Protection, Doctors of the World, Oxfam International.
Dominican Republic gender analysis: study of the impact of the Zika virus on women, girls, boys and men [Internet]. Oxfam International; 2017 [citado 10 mar 2021]. Disponible en: https://tinyurl.com/5dusvjws.

4. Moreira D, Costa MP. The impact of the Covid-19 pandemic in the precipitation of intimate partner violence. International Journal of Law and Psychiatry. 2020;71:101606.

5. Naciones Unidas. Se incrementó un 39\% el pedido de ayuda por violencia de género en Argentina durante el COVID-19 [Internet]. 2020 [citado 11 nov 2020]. Disponible en: https://tinyurl.com/s54vm7as.

6. Observatorio de Femicidios de la Defensoría del Pueblo de la Nación. Femicidios: Informe Anual 2020 [Internet]. Buenos Aires: Defensoría del Pueblo de la Nación; 2020 [citado 10 nov 2020]. Disponible en: https://tinyurl.com/7nexk9vs.

7. Ministerio de las Mujeres, Géneros y Diversidad. Medidas en materia de género y diversidad en el marco de la emergencia sanitaria [Internet]. 2020 [citado $10 \mathrm{mar}$ 2021]. Disponible en: https://tinyurl.com/8yn8rpbk.

8. Tobar F. Impacto del COVID-19 en el acceso a los anticonceptivos en Argentina. Buenos Aires: UNFPA; 2020.

9. Dasgupta A, Kantorová V, Ueffing P. The impact of the COVID-19 crisis on meeting needs for family planning: a global scenario by contraceptive methods used. Gates Open Research. 2020;4(10):1-22.

10. Szulik D, Zamberlin N. La legalidad oculta: Percepciones de estigma en los recorridos de mujeres que descubren y acceden a la interrupción legal del embarazo por causal salud. Sexualidad, Salud y Sociedad. 2020;(37):46-67. 
11. Karstanje M, Ferrari N, Verón Z. El derecho a la salud sexual y reproductiva en Argentina en época de COVID-19. Buenos Aires: Equipo Latinoamericano de Justicia y Género; 2020.

12. Kessler G, coord. Relevamiento del impacto social de las medidas del aislamiento dispuestas por el PEN. Buenos Aires: Ministerio de Ciencia, Tecnología e Innovación; 2020.

13. Braun V, Clarke V. Using thematic analysis in psychology. Qualitative Research in Psychology. 2006;3:77-101.

14. Kapilashrami A, Hankivsky O. Intersectionality and why it matters to global health. The Lancet. 2018;391(10140):2589-2591.

15. Gauthier G, Smith J, García C, Garcia M, Thomas P. Exacerbating inequalities: social networks, racial/ethnic disparities and the COVID-19 Pandemic in the United States. The Journals of Gerontology. 2020;76(3):e88-e92.

16. Burki T. The indirect impact of COVID-19 on women. The Lancet. 2020;20(8):904-905.

17. Sifris R, Penovic T. Barriers to abortion access in Australia before and during the COVID-19 pandemic. Women's Studies International Forum. 2021;86:102470.

18. Drovetta R. Profesionales de la salud y el estigma del aborto en Argentina: El caso de la red de profesionales de la salud por el derecho a decidir. Segunda Época. 2018;(24):13-35

19. Aldavert S, Baroja Benlliure J, Rodríguez García A, Rodríguez Durán A, Tildesley R, Sánchez Torres A. El acceso al aborto en el Estado español: principales barreras. Barcelona: L'Associació de Drets Sexuals i Reproductius; 2020.

20. Tang K, Gaoshan J, Ahonsi B, Ali M, Bonet M, Broutet N, Kara E, Kim C, Thorson A, Thwin SS. Sexual and reproductive health ( $\mathrm{SRH})$ : a key issue in the emergency response to the coronavirus disease (COVID- 19) outbreak. Reproductive Health. 2020;17(1):59.

21. Bateson DJ, Lohr PA, Norman WV et al. The impact of COVID-19 on contraception and abortion health policy and practices: experiences from selected countries. BMJ Sexual \& Reproductive Health. 2020;(46):241-243.

22. Riley T, Sully E, Ahmed Z, et al. Estimates of the potential impact of the COVID-19 pandemic on sexual and reproductive health in low- and middle-income countries. International Perspectives on Sexual and Reproductive Health. 2020;(46):73-76.

23. Socorristas en Red. Sistematización de acompañamientos a abortar realizados en el año 2020 por Socorristas en Red (feministas que abortamos) durante el primer año de pandemia por COVID 19. Comahue: Socorristas en Red; 2021.

24. Usher K, Bhullar N, Durkin J, Gyamfi N, Jackson D. Family violence and COVID-19: Increased vulnerability and reduced options for support. International Journal of Mental Health Nursing. 2020;29(4):549-552.
25. Perez-Vincent SM, Carreras E, Gibbons MA, Murphy TE, Rossi MA, eds. Los confinamientos de la COVID-19 y la violencia doméstica: Evidencia de dos estudios en Argentina. Buenos Aires: Informe Banco Interamericano de Desarrollo; 2020.

26. Boxall H, Morgan A, Brown R. The prevalence of domestic violence among women during the COVID-19 pandemic. Statistical Bulletin. 2000;28:1-28.

27. United Nations Economic and Social Commission for Asia and the Pacific. The Covid-19 Pandemic and violence against women in Asia and the Pacific. Bangkok: United Nations; 2021.

28. Lausi G, Pizzo A, Cricenti C, Baldi M, Desiderio R, Giannini AM, Mari E. Intimate partner violence during the COVID-19 Pandemic: A review of the phenomenon from victims' and help professionals' perspectives. International Journal of Environmental Research and Public Health. 2021;18(12):6204.

29. Williams EE, Arant KR, Leifer VP, Balcom MC, Levy-Carrick NC, Lewis-O'Connor A, Katz JN. Provider perspectives on the provision of safe, equitable, traumainformed care for intimate partner violence survivors during the COVID-19 pandemic: a qualitative study. BMC Womens Health. 2021;21(1):315.

30. van Gelder NE, van Haalen DL, Ekker K, Ligthart SA, Oertelt-Prigione S. Professionals' views on working in the field of domestic violence and abuse during the first wave of COVID-19: a qualitative study in the Netherlands. BMC Health Services Research. 2021;30;21(1):624.

31. Saletti-Cuesta L, Ferioli A, Martínez FDV, Viel E, Baudin V, Romero P, Funk N, González AC, Rodríguez A. El abordaje de la violencia de género desde la perspectiva de las comunidades del norte cordobés, Argentina. Cadernos de Saúde Pública. 2020;36(1):e00184418.

32. Wright EN, Miyamoto S, Richardson C. The impact of COVID-19 restrictions on victim advocacy agency utilization across Pennsylvania. Journal of Family Violence. 2021. doi: 10.1007/s10896-021-00307-z.

33. Regenold N, Vindrola-Padros C. Gender matters: A gender analysis of healthcare workers' experiences during the first COVID-19 pandemic peak in England. Social Science. 2021;10:43.

34. Maffeo F. La incidencia del movimiento feminista en la atención de la salud de mujeres: El caso de los Centros de Atención Primaria de la Salud (CAPS) de Morón. En: Di Marco G, FAySP. Feminismos y populismos del siglo XXI: Frente al patriarcado y al orden neoliberal. Buenos Aires: Teseo; 2019.

35. Rouse LP, Nagy-Agren S, Gebhard RE y Bernstein WK. Women physicians: Gender and the medical workplace. Journal of Womens Health (Larchmt). 2020;29(3):297-309.

36. Saletti-Cuesta L, Aizenberg L, Ricci-Cabello I. Opinions and experiences of primary healthcare providers regarding violence against women. Journal of Family Violence. 2018;33(6):405-420. 
37. Karoff M, Norton C, Tucker, A, Gass, M, Foerster, E. A qualitative gender analysis of women field guides experiences in outdoor behavioral healthcare: a feminist social work perspective. Affilia. 2019;34(1):48-64.

38. Esteban ML. El estudio de la salud y el género: las ventajas de un enfoque antropológico y feminista. Salud Colectiva. 2006;2(1):9-20.
39. Ruiz Pérez I, Pastor Moreno G. Medidas de contención de la violencia de género durante la pandemia de COVID-19. Gaceta Sanitaria. 2021;35(4):389-394.

40. Vives-Cases C, La Parra Casado D, Estevéz J, Torrubiano-Domínguez J, Sanz-Barbero B. Intimate partner violence against women during the COVID-19 lockdown in Spain. International Journal of Environmental Research and Public Health. 2021;18(9):4698.

\section{FORMA DE CITAR}

Saletti-Cuesta L, Aizenberg L. Abordajes de violencias de género y de interrupción legal del embarazo en servicios de salud, durante el aislamiento por COVID-19. Salud Colectiva. 2021;17:e3678. doi: 10.18294/sc.2021.3678.

Recibido: 14 jun 2021 | Versión final: 12 oct 2021 | Aprobado: 2 nov 2021 | Publicado en línea: 17 nov 2021

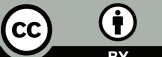

Esta obra está bajo una licencia Creative Commons Atribución 4.0 Internacional (CC BY 4.0). Atribución - Se debe dar crédito de manera adecuada, brindar un enlace a la licencia, e indicar si se han realizado cambios. Puede hacerlo en cualquier forma razonable, pero no de forma tal que sugiera que usted o su uso tienen el apoyo de la licenciante. Sin restricciones adicionales - No se pueden aplicar términos legales ni medidas tecnológicas que restrinjan legalmente a otras personas a hacer cualquier uso permitido por la licencia. 